\title{
Práticas de autocuidado e o papel da enfermagem na assistência ao adulto com Diabetes Mellitus tipo II: um estudo Hondurenho
}

\author{
Self-care practices and nursing role during the care of adults with type II Diabetes Mellitus: a
}

Honduran study

Prácticas de autocuidado y rol de enfermería durante la asistencia al adulto con Diabetes Mellitus

tipo II: un estudio hondureño

Elizabeth Merari Rico Rodríguez

ORCID: https://orcid.org/0000-0001-6742-4308 Universidad Nacional Autonoma De Honduras, Honduras E-mail: elizabeth05rico@yahoo.com

Stefpany Alexandra Santos Jiménez ORCID: https://orcid.org/0000-0002-0112-3956 Universidad Nacional Autonoma De Honduras, Honduras E-mail: stefpany.santos@unah.hn

Eliab Jair Oseguera Oyuela ORCID: https://orcid.org/0000-0002-9847-7979 Universidad Nacional Autonoma De Honduras, Honduras E-mail: eliab.oseguera@unah.edu.hn

Nubia Celeste Rodríguez Matamoros ORCID: https://orcid.org/0000-0001-9707-0977 Universidad Nacional Autonoma De Honduras, Honduras E-mail: nubiacelestero@gmail.com

Jonas Magno dos Santos Cesário ORCID: https://orcid.org/0000-0003-1785-3555 Faculdade Unyleya, Brasil

E-mail: prof.jonasmagno@unyleya.edu.br

Victor Hugo de Paula Flauzino ORCID: https://orcid.org/0000-0001-5156-0030 Faculdade Unyleya, Brasi

E-mail: prof.victorflauzino@unyleya.edu.br

Alexa Merary Cabrera Bucardo ORCID: https://orcid.org/0000-0002-4048-1537 Universidad Nacional Autonoma De Honduras, Honduras E-mail: alexabucardo9@gmail.com

Lourdes Paola Sosa Castellanos ORCID: https://orcid.org/0000-0003-2528-0128 Universidad Nacional Autonoma De Honduras, Honduras E-mail: lourdespaolasosa@gmail.com

Kathia Janahina Melgar Rico ORCID: https://orcid.org/0000-0002-5419-9786 Universidad Nacional Autonoma De Honduras, Honduras E-mail: ricokathia6@gmail.com

Oscar Fidel Antunez Martinez ORCID: https://orcid.org/0000-0002-8301-4795 Universidad Nacional Autonoma De Honduras, Honduras E-mail: ofamice@gmail.com

Judith Victoria Castillo Mejia ORCID: https://orcid.org/0000-0001-5257-6878 Faculdade Unyleya, Brasil

\section{Resumo}

Objetivo: Caracterizar as Práticas de autocuidado de adultos com Diabetes Mellitus tipo 2 (DM tipo 2) e descrever o papel da equipe de enfermagem no cuidado brindado aos pacientes nos diferentes postos de saúde de Tegucigalpa, Honduras. Metodologia: trata-se de um estudo Quantitativo, descritivo, abordagem transversal. Com amostra de 73 adultos de ambos os sexos, selecionados de forma não probabilística por conveniência. A coleta de dados foi realizada com o instrumento eletrônico "Formulários Google", em maio de 2020, utilizando estatística descritiva para análise dos dados. Resultados: foram apresentados na forma de descrição, gráficos e tabelas. Quanto às características 
sociodemográficas: observou-se predomínio do sexo feminino com 59\%. Um 30,6\% relatou receber menos do que o salário mínimo hondurenho (Lps. 8.000,00 -10.000,00). Em relação aos medicamentos: a metformina foi o medicamento mais utilizado (46,6\%). Dentre as medidas de autocuidado, constatou-se que: a maioria dos adultos com DM tipo 2 realiza medicao diária da glicemia; pratica atividades físicas, tem uma alimentação balanceada; verifica seus pés diariamente e considere tomar o tratamento corretamente. Além disso, 94,5\% relataram receber educação para o autocuidado por parte da equipe de enfermagem cada vez que visitam o hospital. Conclusão: a maioria dos adultos com DM tipo 2 estudados, apresentou ações de autocuidado. No entanto, existem lacunas no conhecimento sobre a doença e no uso correto dos medicamentos. Nesse sentido, considera-se de vital importância, o papel da enfermagem na educação para a saúde desta população.

Palavras-chave: Diabetes Mellitus tipo 2; Autocuidados; Adulto; Assistência de enfermagem.

\begin{abstract}
Objective: To characterize the self-care practices of adults with Type 2 Diabetes Mellitus (DM type 2) and to describe the role of the nursing staff during the care of these patients in different health care centers in Tegucigalpa, Honduras. Methodology: Quantitative, descriptive, cross-sectional approach, with a sample of 73 adults of both sexes, selected non-probabilistically by convenience. Data collection was carried out with the electronic instrument "Google Forms", in May 2020, using descriptive statistics for data analysis. Results: these were presented in the form of descriptions, graphs, and tables. Concerning the sociodemographic characteristics: the predominance of the female sex was observed with 59\%. The 30.6\% reported receiving less than the Honduran minimum wage (Lps. $8000.00-10,000.00)$. Regarding medication: Metformin was confirmed to be the most used medication (46.6\%). Among the self-care measures, it was found that: most adults with type 2 DM perform daily blood glucose level controls; engage in physical activity and eat a balanced diet; check their feet daily and consider taking the treatment correctly. In addition, $94.5 \%$ reported receiving education on self-care by the nursing staff every time they go to the hospital. Conclusion: most of the adults with type 2 DM studied reported performing self-care actions. However, there are gaps in knowledge about the disease and the correct use of medications. Therefore, the role of nursing in the health education of this population is of vital importance. Keywords: Diabetes Mellitus type 2; Self-care; Adult; Nursing care.
\end{abstract}

\title{
Resumen
}

Objetivo: Caracterizar las prácticas de autocuidado del adulto con Diabetes Mellitus Tipo 2 (DM tipo 2) y describir el rol del personal de enfermería durante la asistencia a estos pacientes en los diferentes centros asistenciales de Tegucigalpa, Honduras. Metodología: Enfoque cuantitativo, descriptivo, transversal, con una muestra de 73 personas adultas de ambos sexos, seleccionados de forma no probabilística por conveniencia. La recolección de datos se realizó con el instrumento electrónico "Google Forms", en el mes de mayo, 2020, utilizando la estadística descriptiva para el análisis de datos. Resultados: estos fueron presentados en forma de descripción, gráficos y tablas. Con relación a las características sociodemográficas: se observó el predominio del sexo femenino con 59\%. El 30.6\% reportó recibir menos del salario mínimo hondureño (Lps. 8000.00 -10,000.00). Con relación a la medicación: La metformina confirmó ser el medicamento más utilizado (46.6\%). Entre las medidas de autocuidados se encontró que: la mayoría de los adultos con DM tipo 2 realiza controles diarios del nivel de glucosa sanguínea; realiza actividad física y consumo de dieta equilibrada; revisa diariamente sus pies y considera tomar el tratamiento de forma correcta. Además, un $94.5 \%$ refirió recibir educación sobre autocuidados por el personal de enfermería cada vez que acude al hospital. Conclusión: la mayoría de los adultos con DM tipo 2 estudiados, reveló realizar acciones de autocuidado. Sin embargo, existen lagunas de conocimiento sobre la enfermedad y el uso correcto de los medicamentos. Por lo que es de vital importancia, el papel de enfermería en la educación en salud a esta población.

Palabras clave: Diabetes Mellitus tipo 2; Autocuidado; Adulto; Atención de enfermería.

\section{Introdução}

O diabetes mellitus do tipo II (DM2), é considerado um distúrbio metabólico caracterizado por hiperglicemia crônica devido a uma diminuição da secreção de insulina. Ela surge quando o organismo não consegue controlar a quantidade de glicose no sangue, que ocorre se o organismo não produz insulina suficiente para metabolizar o açúcar no sangue. A insulina é reconhecida como um hormônio que ajuda a glicose a entrar nas células para obter energia. Sem insulina, a glicose no sangue aumenta. Este aumento da glicemia, ao longo do tempo, danifica gravemente muitos órgãos e sistemas, especialmente nervos e vasos sanguíneos (OMS, 2021)

Globalmente, estima-se que 346 milhões de pessoas tenham diabetes, e espera-se que este número duplique até 2030. Na Região das Américas, estima-se que 62 milhões de pessoas têm DM2, e se as tendências atuais continuarem, espera-se que este número aumente para 91,1 milhões até 2030 (OPS, 2017; American Diabetes Association, 2014). 
O diabetes mellitus é uma das quatro doenças não transmissíveis mais comuns na população e, de alguma forma, é fatal. Em Honduras, estima-se que a taxa de mortalidade por diabetes seja de 2\% para o ano de 2018. Da mesma forma, esse país ocupa o terceiro lugar na prevalência de diabetes na América Central com uma taxa de 9,53\% (Sánchez, 2018).

A prevalência dessa patologia nos países ocidentais é de 2-6\% da população adulta, sendo o DM2 a forma mais comum, com uma proporção de 9:1 para o diabetes tipo 1. Pesquisas sobre diabetes mostram que mais da metade dos pacientes manifestam estilos de vida pouco saudáveis, como o não cumprimento da dieta e da atividade física, falta de controle metabólico, abuso da ingestão de substâncias psicotrópicas, bem como conhecimento insuficiente sobre sua doença (Rivera \& Lucio, 2018).

Neste sentido, a educação em autocuidado é reconhecida como um elemento crítico no processo de cuidados para pacientes com DM2 e aqueles em risco de desenvolver uma doença crônica (Poma, 2015). Assim, nesse estudo, objetivou-se caracterizar as práticas de autocuidado de adultos com DM2 e descrever o papel da equipe de enfermagem no cuidado brindado aos pacientes nos diferentes postos de saúde de Tegucigalpa, Honduras.

\section{Metodologia}

Este é um estudo quantitativo, descritivo, com um alcance transversal, pois aborda variáveis dependentes tais como: histórico de diabetes, medidas de autocuidado, entre outras (Cesario, Flauzino \& Mejia, 2020; Estrela, 2018). A coleta de dados foi realizada virtualmente usando o instrumento eletrônico Google Forms, durante os meses de março a maio de 2020.

A população de interesse eram adultos a partir dos 35 anos com diabetes mellitus tipo II que vivessem na cidade de Tegucigalpa, Honduras. A amostra foi não-probabilística, onde os participantes foram selecionados por conveniência e de forma aleatória. A amostra final consistiu de 73 adultos de ambos os sexos.

Para a coleta de dados foi utilizada uma técnica de levantamento com 21 perguntas estruturadas e fechadas, focadas em medidas de autocuidado, conhecimento da patologia. Algumas das variáveis incluídas foram: sexo, idade, histórico de diabetes mellitus tipo II, medidas de autocuidado para diabetes mellitus, tipo de medicação utilizada, frequência de controles médicos, frequência de controles do nível de glicose, importância dos cuidados com os pés, entre outras. Cada pergunta foi explicada de uma maneira clara e fácil. Para a análise dos dados, foram utilizadas estatísticas descritivas, o que facilitou a apresentação dos resultados na forma de descrições, gráficos e tabelas para uma melhor compreensão.

\section{Resultados e Discussão}

\section{Caraterísticas sociodemográficas}

O Gráfico 1 apresenta os resultados da pesquisa, correspondente a variável "sexo".

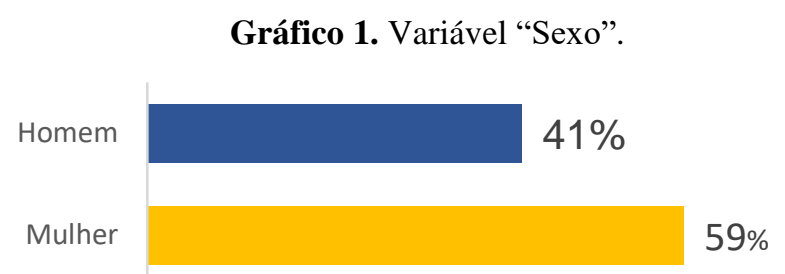

Fonte: Dados extraídos do instrumento eletrônico Google Forms, elaborado pelos autores (2021).

Os resultados obtidos são semelhantes aos estudos realizados por diferentes autores no Peru, onde mostram que a população feminina nesta faixa etária é a mais afetada pela DM2. Ainda de acordo com o sexo, foi observada uma prevalência do diabetes no sexo feminino. Nesse contexto, encontrou-se que os principais fatores associados foram a obesidade e um estilo 
de vida sedentário, com predominância das mulheres, tendo com isso um aumento do risco de sofrer de diabetes mellitus tipo 2 (Quimí \& Stalin, 2018).

O Gráfico 2 apresenta os resultados da pesquisa, correspondente a variável "idade”. Assim, aprecia-se que as idades dos participantes eram de 35 até 65 anos, conforme gráfico seguinte.

Gráfico 2: variavel "Idade".

\section{Idade}

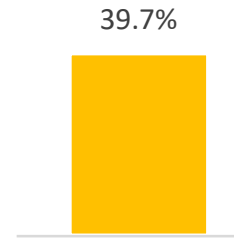

A- $35-45$

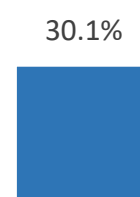

B- $46-55$
$30.1 \%$

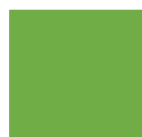

C- $56-65$

Fonte: Dados extraídos do instrumento eletrônico Google Forms, elaborado pelos autores (2021).

Em se tratando da variável idade, é importante notar que nessa pesquisa, a maioria dos pacientes com DM tipo 2, encontra-se entre 35-45 anos de idade, sendo a maioria, feminino. Nesse sentido, estudos apontam que também existe um aumento significativo na prevalência do DM tipo 2 nas idades de 50 a 59 anos, coincidindo com o que foi descrito na Pesquisa Nacional de Saúde e Nutrição do Equador de 2013, onde este aumento também foi observado a partir do mesmo grupo etário (10,3\% versus 5,4\% no grupo de 40 a 49 anos). A idade mais avançada é universalmente reconhecida como um dos principais fatores de risco para o DM tipo 2, em associação com fenômenos como a disfunção pancreática das células beta, senescência celular generalizada, aumento da produção de metabólitos tóxicos como produtos avançados de glicação e espécies reativas de oxigênio (Freire et al. 2013; Altamirano Cordero et al. 2017).

O grupo de adultos com mais de 59 anos, é considerado vulnerável na maioria dos países, não só devido às condições inerentes ao processo de envelhecimento, mas também pelas desvantagens socioeconômicas, pois muitos indivíduos atingem essa idade sem apoio financeiro garantido (pensão) e sem apoio familiar o suficiente. Este último fator é explicado por mudanças substanciais sofrido por famílias urbanas, onde membros maiores de idade devem ir trabalhar, saindo desprotegido dos cuidados necessários para adultos mais velhos que deveriam ficar em casa (García et al. 2014; Rodrigues et al. 2014; Manoel et al. 2015; Sánchez \& Medina, 2015; Imazu et al. 2015).

O Gráfico 3 apresenta os resultados da pesquisa, correspondente a variável "Renda familiar". 
Gráfico 3: Variável "Renda familiar".

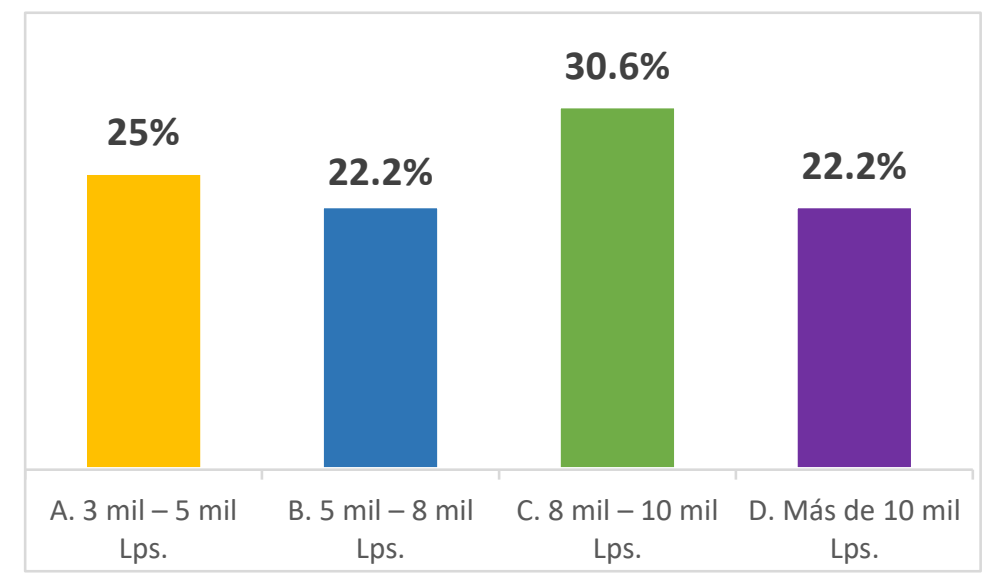

Fonte: Dados extraídos do instrumento eletrônico Google Forms, elaborado pelos autores (2021).

Em relação ao nível de renda dos participantes com diabetes mellitus tipo 2, observa-se que 30,6\% dos entrevistados recebem entre 8,000 - 10,000 lempiras-lps (o salário mínimo hondurenho em 2019 era de 9.443,24 Lps), ou seja, que menos da metade dos entrevistados tem ingresos económicos na faixa do salário mínimo hondurenho. $25 \%$ da população entrevistada vive com menos da metade do salário mínimo (3,000-5,000 Lps), 22,2\% vive com 5,000-8,000 Lps e 22,2\% dos participantes têm um rendimento económico superior a 10 mil Lps.

Um estudo realizado na Colômbia mostrou que os adultos com maior renda familiar tinham mais acesso aos serviços de saúde e menos risco de sofrer depressão ou ansiedade. No entanto, todos eles sofriam de limitações para realizar atividade física, fator determinante para um estilo de vida saudável que favorece o controle da doença (Alpizar, Trujillo, Gutiérrez \& Sánchez, 2017).

O Gráfico 4 apresenta os resultados da pesquisa, correspondente a variavel "Antecedentes de acordo com o Diabetes Mellitus tipo 2".

Gráfico 4: Variavel "Antecedentes de acordo com o DM2".

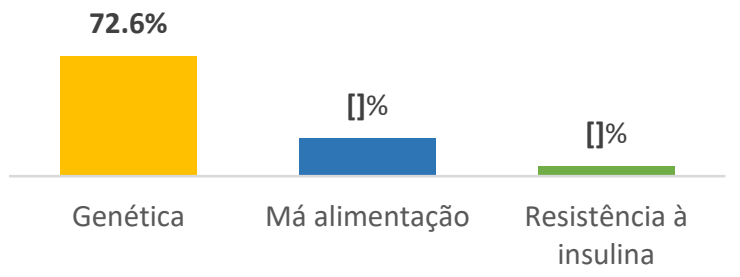

Fonte: Dados extraídos do instrumento eletrônico Google Forms, elaborado pelos autores (2021).

O DM tipo 2 diversas etiologias. Assim, nessa pesquisa, prevaleceram mais os antecedentes genéticos para o desenvolvimento dessa patologia. Pode-se observar que a grande maioria dos pacientes/usuários (72.6\%) tinham antecedentes familiares de diabetes. Embora a genética seja um fator no desenvolvimento do diabetes, essa não determina o aparecimento da doença. Algumas pessoas nascem com uma predisposição para a doença, mas fatores ambientais são necessários para desencadear a doença (López, 2019).

Foi evidenciado que a genética e história familiar jogam um papel importante no desenvolvimento da DM tipo 2. Nesse sentido, estudos apoiam esse fato e ainda confirmam que aunado a isso, os maus hábitos alimentares poderiam desencadear a 
resistência a insulina. Esta resistência à insulina faz com que as células do organismo não respondam à insulina, aumentando com isso a glicemia na sangue (Agüero, Piña \& Pérez, 2012; Valdés, Hernández \& Ferrer, 2015).

O Gráfico 5 apresenta os resultados da pesquisa, correspondente a variável "A equipe de enfermagem fornece informações sobre autocuidado?"

Gráfico 5: Variável “A equipe de enfermagem fornece informações sobre autocuidado?”.

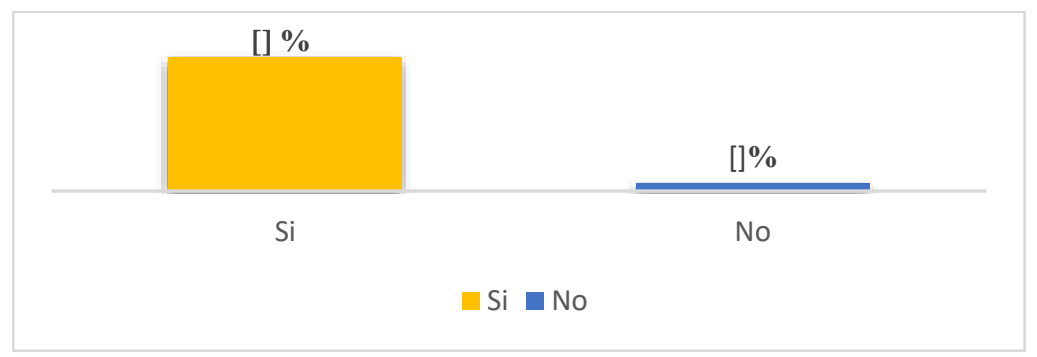

Fonte: Dados extraídos do instrumento eletrônico Google Forms, elaborado pelos autores (2021).

Observa-se que 94,5\% dos pesquisados afirmam ter recebido por parte da equipe de enfermagem informações sobre o autocuidado que devem ter frente a doença. Em relação ao nível de conhecimento sobre DM tipo 2, os resultados coincidem com investigações nas quais os pacientes não apresentam dificuldades em reconhecer os fatores de risco, as medidas de autocuidado, bem como os seus respectivos tratamentos. A este respeito, o conhecimento sobre a patologia influencia num melhor controle metabólico. Embora o conhecimento não seja o único aspecto a ser considerado para a modificação do comportamento, é transcendente desenvolver ações preventivas e comportamentos de autocuidado. Nesse contexto, um estudo realizado em Cuba, mostrou que $94 \%$ dos usuários/pacientes asseguram ter informações sobre o autocuidado de acordo com seu diagnóstico. Ressaltando que a falta de informação é lesiva a longo e curto prazo (Sánchez et al. 2016; Alpizar, Trujillo, Gutiérrez \& Sánchez, 2017).

\section{Papel da equipe de enfermagem na assistência ao paciente diabético}

O profissional de enfermagem envolve uma visão abrangente do paciente e de seu contexto. As competências desenvolvidas por meio da educação permanente capacitam o enfermeiro na oferta de uma assistência de qualidade, como benefícios garantidos. As intervenções educativas realizadas durante o exame de saúde também fazem parte desse fator. Reconhece-se a necessidade de realizar programas educacionais formais (oficinas de educação em grupo) e informais (intervenções educativas no controle da saúde) para evitar complicações causadas pelo desconhecimento (Gomes et al. 2021).

Nesse sentido, tanto as intervenções educativas grupais quanto individuais têm se mostrado eficazes e são mais benéficas quando utilizam meios ou estratégias que favorecem a construção do conhecimento, ao invés da transmissão de realizada no modelo tradicional. Assim, é importante aproximar-se de um entendimento dialógico de saberes e eliminar a transmissão de informações de forma unidirecional, uma vez que esta tem se mostrado insuficiente no autocuidado do paciente, e mesmo na formação profissional. Por tanto, essa intervenção tem de ser orientada para uma educação integral que promova a compensação metabólica e o bem-estar das pessoas que sofrem de DM tipo 2 (Alpizar, Trujillo, Gutiérrez \& Sánchez, 2017).

\section{Práticas de autocuidado de adultos com diabetes mellitus tipo 2}

O Quadro 1 a seguir, apresenta os resultados da pesquisa, correspondente a variável "Medidas de autocuidado tomadas pelos participantes com relação ao DM2". 
Quadro 1. Variável "' Medidas de autocuidado tomadas pelos participantes com relação ao DM2".

\begin{tabular}{|l|l|}
\hline Medidas de autocuidado & Valor percentual \\
\hline Variável & $24,6 \%$ \\
\hline Alimentação saudável & $23,7 \%$ \\
\hline Tratamento da maneira correta & $10,1 \%$ \\
\hline Verificação dos pés & $13,6 \%$ \\
\hline Atividade física & $28,0 \%$ \\
\hline Todas as anteriores & 100 \\
\hline Total & \\
\hline
\end{tabular}

Fonte: Dados extraídos do instrumento eletrônico Google Forms, elaborado pelos autores (2021).

Observa-se que a maioria relatou realizar medidas de autocuidado como ter uma dieta saudável, tem uma forma correta de tratamento, cuidado apropriado dos pés e exercício. Nesse contexto, o autocuidado é entendido como um componente fundamental no manejo do tratamento em pessoas que sofrem de DM, pois exige a aquisição de conhecimentos e habilidades dos pacientes. Neste sentido a OMS tem sugerido a educação sobre o autocuidado para permitir ao paciente ser protagonista no seu tratamento. Assim, pessoas com DM tipo 2, devem realizar técnicas de autocuidado como alimentação saudável, atividade física, glicometria e uso adequado dos medicamentos indicados (Oliveira, Costa, Ferreira \& Lima, 2017).

Entendesse que uma vez reconhecido o caso clínico, o paciente deve ser informado sobre sua doença para conhecer as medidas eficientes de autocuidado que permitam levar um estilo de vida saudável. Destacasse também que o plano alimentício dependerá da idade, sexo, estado nutricional e atividade física além do estado fisiológico, patológico e seus níveis de glicose (Baquedano, Santos, Martins \& Zanetti, 2010).

O Gráfico 6 a seguir, apresenta os resultados da pesquisa, correspondente a variavel "Tipo de medicamento utilizado".

Gráfico 6: Variável "Tipo de medicamento utilizado".

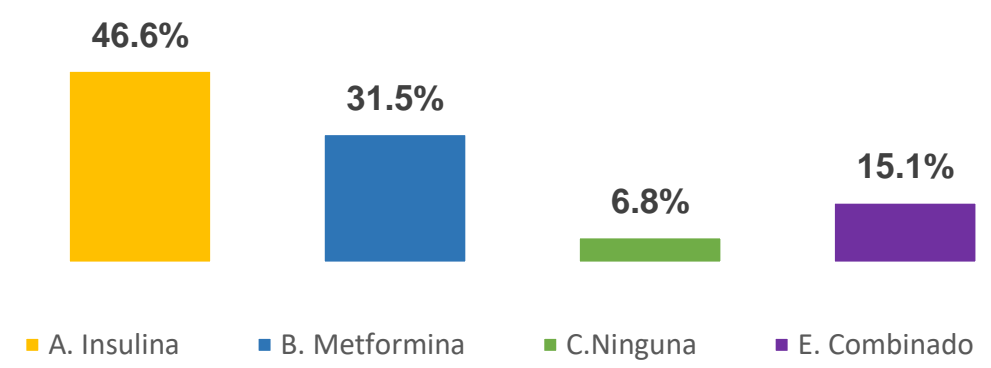

Fonte: Dados extraídos do instrumento eletrônico Google Forms, elaborado pelos autores (2021).

Os adultos com maior idade seguem o tratamento farmacológico indicado, sendo que 46,6\% deles usam a insulina e $31,5 \%$ a Metformina, 6,8\% não usam nenhum desses medicamentos, e 15,1\% usam tratamento combinado (Insulina/comprimido Oral). Da mesma forma, revelou-se num estudo, que a adesão ao tratamento farmacológico é um fator fundamental no controle do diabetes mellitus, permitindo a manutenção e recuperação da saúde dos usuários. No estudo mencionado, 51,7\% dos adultos idosos com DM tipo 2 aderiram-se ao tratamento farmacológico e 48,2\% não seguiram o tratamento prescrito (Escamilla \& Montoya, 2017). 
Segundo a OPAS, os adultos mais velhos consomem o dobro de medicamentos que os mais jovens. Por este motivo, é importante obter um histórico de medicamentos, incluindo alergias, reações adversas, drogas auto prescritas e questionários sobre vícios como álcool, tabaco e cafeína. Alguns fatores que interferem no tratamento são: nível socioeconômico, diminuição da acuidade visual, audição, tato e olfato, portanto, o envolvimento da família no tratamento do paciente também é muito importante (OPS, 2017).

O Gráfico 7 a seguir, apresenta os resultados da pesquisa, correspondente a variavel "Frequência das consultas médicas de acompanhamento"

Gráfico 7: Variável "Frequência das consultas médicas de acompanhamento".

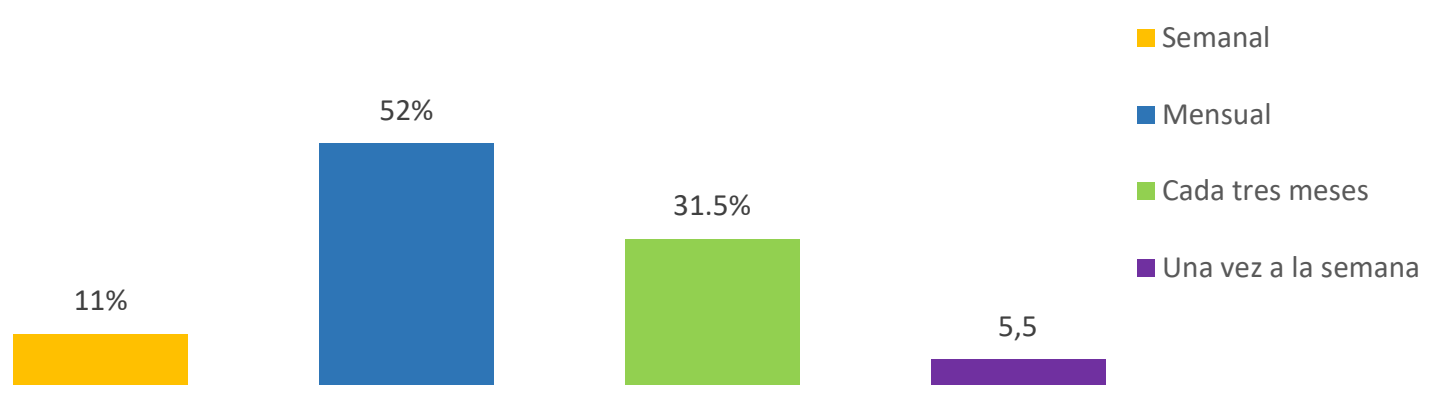

Fonte: Dados extraídos do instrumento eletrônico Google Forms, elaborado pelos autores (2021).

Observou-se que a grande maioria (52\%) dos pacientes têm consultas mensais, 31,5\% têm a cada três meses, $11 \%$ semanais e somente uma minoria (5,5\%) uma vez por semana. Esses dados mostram que os pacientes estão cientes da doença e dos valores glicêmicos e, portanto, visitam regularmente o médico. Nestas visitas realizam o controle de rotina dos níveis de glicose onde seu autocuidado é evidenciado pela melhoria e controle de sua saúde. Esses resultados coincidem com um estudo que afirma que a educação para o DM2 deve ser priorizada na intervenção psicológica e que entre os tópicos a serem desenvolvidos estão o enfrentamento da patologia, melhorando a adesão ao tratamento e o controle glicêmico, aspectos que estão relacionados ao autocuidado (Rivera \& Lucio, 2018).

Além disso, é bem conhecido o quanto importante é o conhecimento que os pacientes com DM tipo 2 devem ter sobre sua patologia para o autocuidado. Em um estudo realizado no Peru, apenas 38,7\% dos participantes estudados tinham um bom conhecimento do que caracteriza a doença, uma proporção menor do que na população analisada. Também, em um estudo do México, foi demonstrado que $42,4 \%$ dos pacientes tinham conhecimento dos níveis de glicose no sangue necessarios para serem diagnosticados com DM2, bem como para comparecer a consultas hospitalares ou consultas medicas de seguimento (Ortega et al. 2012).

A seguir, apresenta-se os resultados da pesquisa, correspondente a variavel "Controles dos níveis de glicose no sangue" conforme Quadro 2. 
Quadro 2: Variável "Controles dos níveis de glicose no sangue".

\begin{tabular}{|l|l|}
\hline Controles dos níveis de glicose no sangue & \\
\hline Variável & Valor percentual \\
\hline Todos os dias & $31,1 \%$ \\
\hline Por Semana & $29,5 \%$ \\
\hline A cada duas ou três semanas & $11,5 \%$ \\
\hline Todo mês & $11,5 \%$ \\
\hline Quando sente um dos sintomas associados & $11,5 \%$ \\
\hline Somente quando vai uma outra consulta médica & $4,9 \%$ \\
\hline
\end{tabular}

Fonte: Dados extraídos do instrumento eletrônico Google Forms, elaborado pelos autores (2021).

O $31,1 \%$ dos participantes responderam que verificam sua glicose todos os dias, um $29,5 \%$ todas as semanas, $11,5 \%$ a cada duas a três semanas, assim como $11,5 \%$ a cada mês, igualmente os que verificam sua glicose quando se sentem algum sintoma associado, e 4,9\% verificam sua glicose somente quando vão a uma consulta médica. O controle adequado da glicose ajuda a ter um melhor controle da doença e consequentemente prevê um bom prognóstico do paciente. Nesse sentido, a OMS menciona a relevância de testar a hemoglobina glicosilada (HbA1C) a fim de orientar as decisões de tratamento. Este teste sanguíneo (HbA1C) é utilizado para conhecer o controle metabólico do açúcar no sangue nos 2 ou 3 meses anteriores (OMS, 2021).

Por outro lado, observou-se em um estudo realizado no Equador que 64,1\% dos pacientes com 5 anos ou mais de diagnóstico, tinham um controle inadequado da doença, mostrando com isso a perca de interesse no autocuidado da doença com o passar do tempo (Luna, 2018).

No gráfico seguinte, apresenta-se os resultados da pesquisa, correspondente a variavel "Importância do cuidado dos pés" conforme Gráfico 8.

Gráfico 8: Variável "Importância do cuidado dos pés".

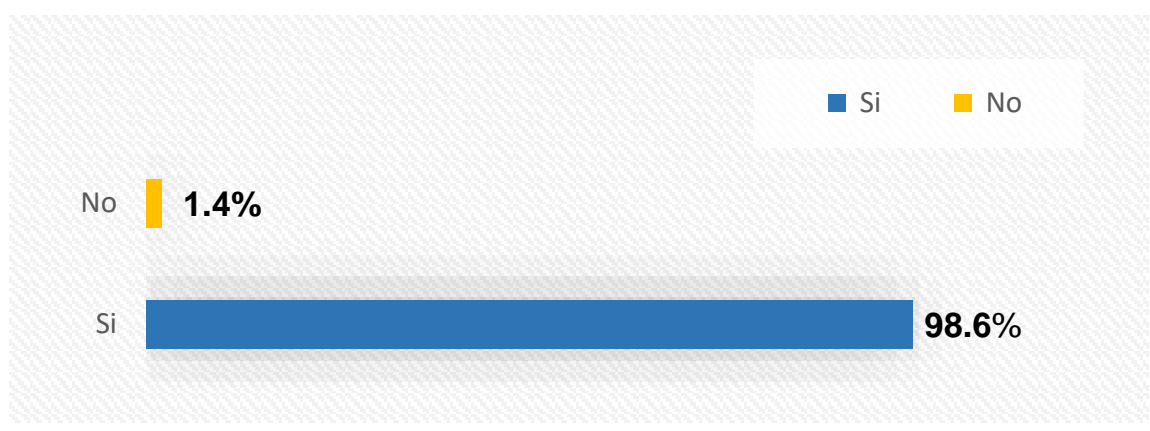

Fonte: Dados extraídos do instrumento eletrônico Google Forms, elaborado pelos autores (2021).

Neste gráfico observa-se que a grande maioria $(98,6 \%)$, consideram o cuidado dos pés de uma pessoa diabética, como uma atividade imprescindível na rutina de autocuidado. Este dado concorda com os resultados de um estudo realizado no Brasil, onde o 98,3\% dos indivíduos estudados reportou-se como cientes da importância do cuidados dos pés para prevenir complicações, como o pé diabético. Dentre dessas atividades, relataram a secagem dos pés, a secagem da sola, costas dos pés e entre os dedos dos pés, como um hábito que visa prevenir a proliferação de microrganismos e causar rachaduras na pele (Silva et al. 2015). 
A seguir, apresenta-se os resultados da pesquisa, correspondente a variável "Qual a importância do cuidado dos pés" conforme Quadro 3.

Quadro 3: Variável "Qual a importância do cuidado dos pés".

\begin{tabular}{|l|l|}
\hline Variável & Valor percentual \\
\hline Prevenir o pé diabético. & $65.8 \%$ \\
\hline As unhas podem ficar encravadas & $1.3 \%$ \\
\hline Cogumelos podem crescer. & $11 \%$ \\
\hline Todas as anteriores & $21.9 \%$ \\
\hline Total & $100 \%$ \\
\hline
\end{tabular}

Fonte: Dados extraídos do instrumento eletrônico Google Forms, elaborado pelos autores (2021).

Observou-se que a grande maioria (65.8\%), considera o cuidar dos pés como a melhor forma de prevenir o pé diabético, complicação muito comum em um paciente com esta patologia (Paiva \& Rojas 2016; Solano \& Saénz, 2021).

\section{Conclusão}

Verificou-se que a maioria dos participantes são do sexo feminino e entre os 35-45 anos de idade. Nesse estudo foi possível identificar as práticas de autocuidado, sendo possível distinguir o conhecimento que o adulto maior tem sobre sua patologia. Os dados indicaram que os participantes são cientes da importância de cuidar de seus pés para evitar complicações. Vê-se a importância do papel dos profissionais de enfermagem, pois estes sãos responsáveis da intervenção educativa nesses usuários/pacientes, visando garantir a adesão ao tratamento e com isso um melhor prognostico da doença e consequentemente qualidade de vida.

Recomenda-se o desenvolvimento de mais pesquisas sobre o autocuidado dos pacientes com DM tipo 2, para aprofundar no conhecimento, e desenvolver planos de intervenções baseado em evidencias.

\section{Referências}

Agüero, S. D., Piña, E. C. \& Pérez, M. A. (2012). Alimentación y Diabetes. Nutrición Hospitalaria. 27. https://scielo.isciii.es/scielo.php?script=sci_a rttext\&pid=S0212-16112012000400010.

Alpizar, E. M. R., Trujillo, G. Z., Gutiérrez, C. H. \& Sánchez, B. V. (2017). Manejo práctico del paciente con diabetes mellitus en la Atención. Primaria de Salud. Revista Finlay. 1 (3). https://www.medigraphic.com/pdfs/finlay/fi-2017/fi171n.pdf.

Altamirano-Cordero, L.C., Vásquez, M. A., Cordero, G., Álvarez, R., Añe, R., Rojas, J. \& Bermúdez, V. (2017): Prevalencia de la diabetes mellitus tipo 2 y sus factores de riesgo en individuos adultos de la ciudad de Cuenca- Ecuador. Avan Biomed. 6(1): 10-21.

American Diabetes Association. (2014). Diagnosis and Classification of Diabetes Mellitus. Diabetes Care. 37 : 81-90.

Baquedano, R. R., Santos, M. A., Martins, T. A. \& Zanetti, M. L. (2010). Autocuidado de personas con Diabetes Mellitus atendidas en un servicio de urgencia en México. Rev. Latino-Am. Enfermagem 18(6). https://www.scielo.br/j/rlae/a/VCzrRDgHSY5HcKMzxhgtHXB/?format=pdf\&lang=es.

Cesário, J. M. S., Flauzino, V. H. P. \& Mejia, J. V. C. (2020). Metodologia científica: Principais tipos de pesquisas e suascaraterísticas. Revista Científica Multidisciplinar Núcleo do Conhecimento. 5(11), 23-33. https://doi.org/10.32749/nucleodoconhecimento.com.br/educacao/tipos-de-pesquisas.

Escamilla, N. V. B. \& Montoya, P. A. C. (2017): Adherencia al tratamiento farmacológico en adultos mayores diabéticos tipo 2 y sus factores asociados. Gerokomos. 28 (2), 73-77. https://scielo.isciii.es/scielo.php?script=sci_abstract\&pid=S1134-928X2017000200073.

Estrela, C. (2018). Metodología científica: ciencia, docencia, investigación. Editorial de Artes Médicas.

Freire, W. B., Ramírez, M. J., Belmont, P., Mendieta, M. J., Silva, K. M. \& Romero, N. (2013). Encuesta Nacional de Salud y Nutrición. Ecuador. https://www.ecuadorencifras.gob.ec/documentos/web-inec/Estadisticas_Sociales/ENSANUT/Publicacion\%20ENSANUT\%202011-2013\%20tomo\%201.pdf. 
García, C., Cruz, E., Gómez, D., Toxqui, M. \& Sosa, B. (2014). La percepción de un grupo de hombres sobre la diabetes mellitus: contribuciones a la enfermería. Esc Anna Nery. 18(4):562-9.

Gomes, D. M., Mejía, J. V. C., Vitorino, P. G. S., Ribeiro, D. V., Hernandes, L. O., Lima, T. O. P., Chã, N. V., Flauzino, V. H. P., Cusato, T. V., \& Cesário, J. M. S. (2021). Educação digital na formação de profissionais de saúde. Research, Society and Development. 10(8), e4110816885. https://doi.org/10.33448/rsdv10i8.16885.

Imazu, M., Faria, B., Arruda, G., Sales, C. \& Marcon, S. (2015). Efectividad de las intervenciones individual y en grupo en personas con diabetes tipo 2. Rev. Latino-Am. Enfermagem. 23(2):200-7.

López, J. I. (2019). Factores presentes en diabéticos tipo II que asisten al programa de dispensarizados del puesto de salud Antenor Sandino Hernández, LeónNicaragua II semestre 2018 [Tesis]. Universidad Nacional Autónoma de Nicaragua, Managua, Nicaragua. https://repositorio.unan.edu.ni/11079/1/t1064.pdf

Luna, E.V.C. (2018). Prevalencia y factores asociados al control glicémico en pacientes con Diabetes Mellitus Tipo 2. Hospital José Carrasco Arteaga. Cuenca, 2016-2017. [Tesis]. Universidad de Cuenca, Cuenca. http://dspace.ucuenca.edu.ec/handle/123456789/30430.

Manoel, M., Nascimiento, B., Oliveira, G., Aparecida, C. \& Silva, S. (2015). Efectividad de las intervenciones individual y en grupo en personas con diabetes tipo 2. Rev. Latino-Am. Enfermagem. 23(2):200-7.

Oliveira, P. S., Costa, M. M., Ferreira, J. D. L. \& Lima, C.L.J. (2017). Autocuidado en Diabetes Mellitus: estudio bibliométrico. Enfermería Global (45). https://scielo.isciii.es/pdf/eg/v16n45/1695-6141-eg-16-45-00634.pdf.

OMS (2021). Diabetes. https://www.who.int/es/news-room/fact-sheets/detail/diabetes.

OPS (2017). Diabetes. OPS, Washington. https://www3.paho.org/hq/index.php?option=com_content\&view=category\&layout=blog \&id=4475\&Itemid=4 0610\&lang=es.

Organización Mundial de la salud (OMS). (2021). Diabetes: Datos y cifras. 2021. OMS, https://www.who.int/es/news-room/fact-sheets/detail/diabetes.

Ortega, M. R. O., Domínguez, M. A. D., Moreno, M. C. C., Sierra, M. S. \& Porras, M. (2012). Estrategia de intervención educativa en adultos mayores diabéticos tipo 2. Rev. Ciencias Médicas. 16(3):109-119. https://www.medigraphic.com/pdfs/pinar/rcm-2012/rcm123k.pdf.

Paiva, O. \& Rojas, N. (2016). Diabetes. Revista Médica Clínica Las Condes. 2016. 27 (2), pág. 227-234. https://www.elsevier.es/es-revista-revista-medicaclinica-las-condes-202-articulo-pie-diabetico-podemos-prevenirlo-S0716864016300128.

Poma, K. L. T. (2015). Nivel de conocimiento y su relación con la práctica del autocuidado en adultos con diabetes Mellitus tipo II, en el CS. San Francisco Tacna 2015. [Tesis]. Universidad Nacional Jorge Basadre Grohmann, Tacna- Perú. http://repositorio.unjbg.edu.pe/bitstream/handle/UNJB G/2175/729_2015_tuyo_poma_kl_facs_enfermeria.pdf?sequence=1\&isAllowed=y.

Quimí, A. \& Stalin, C. (2018): Factores de riesgo asociados a diabetes mellitus tipo II en el Subcentro de Salud Vergeles. [Tesis]. Universidad de Guayaquil, Guayaquil. http://repositorio.ug.edu.ec/handle/redug/30747.

Rivera, K. J. R. \& Lucio, M. E. V. (2018). Autocuidado de la Diabetes Mellitus tipo 2 en adultos de 40-60 años atendidos en la consulta externa del Hospital General IEES Ceibos. [Tesis]. Universidad Católica de Santiago de Guayaquil, Guayaquil. 2018. http://repositorio.ucsg.edu.ec/handle/3317/11181.

Rodrigues, F., Dias, F., Zuffi, F., Borgues, M., Lara, B. \& Ferreira L. Cuidado de los pies: conocimiento de los individuos con diabetes mellitus. Enferm Global. 13(35):41-51.

Sánchez, R. (2018). Honduras ocupa el tercer lugar en prevalencia de diabetes en Centroamérica. El Heraldo. https://www.elheraldo.hn/pais/1234628466/honduras-ocupa-el-tercer-lugar-en-prevalencia-de-diabetes-en-centroam\%C3\%A9rica.

Sánchez, S. \& Medina, J. La interacción entre la perspectiva epistemológica de las enfermeras educadoras y los participantes (en programas educativos): límites y oportunidades en el desarrollo del empoderamiento para el fomento del autocuidado en salud. Texto Contexto Enferm. 2015;24(2):301-9.

Sánchez, Y. M. S., Rosabal, E. P., Sánchez, M. C. L. \& Rodríguez, D. Q. (2016). Conocimientos y autocuidado en pacientes con diabetes mellitus tipo 2. Revista Archivo Médico de Camagüey. 20 (3). http://scielo.sld.cu/scielo.php?script=sci_arttext\&pid=S1025-02552016000300004.

Silva, L. P., Rezende, M. P., Ferreira, L. P., Dias, F. A., Helmo, F. R. \& Silveira, F. O.C. (2015). Cuidados de los pies: el conocimiento de las personas con diabetes mellitus inscritos en el programa de salud familiar. Enfermería Global. 14(37), 38-51. 14. https://scielo.isciii.es/scielo.php?script=sci_a rttext\&pid=S1695-61412015000100003.

Solano, A. A. L. \& Saénz, N. R. T. (2021): Conocimientos, actitudes y prácticas sobre pie diabético en pacientes con Diabetes Mellitus tipo 2 atendidos en el Hospital Primario Bello Amanecer, I Semestre del 2020. [Tesis]. Universidad Nacional Autónoma de Nicaragua, https://repositorio.unan.edu.ni/14962/1/14962.pdf.

Valdés, M. T. L., Hernández, I. L. \& Ferrer, R. L. (2015): Interacción genoma-ambiente en la aparición de la Diabetes Mellitus tipo 2 en una población del municipio Plaza de la Revolución. Revista Cubana de Investigaciones Biomédicas. 34(4), 294-312. http://scielo.sld.cu/scielo.php?script=sci_arttex $\mathrm{t} \&$ pid=S0864-03002015000400001\&lng=es\&tlng=es. 